Review

\title{
Dynamic purine signaling and metabolism during neutrophil-endothelial interactions
}

\author{
Thomas Weissmuller ${ }^{1}$, Holger K. Eltzschig ${ }^{1,2}$ \& Sean P. Colgan ${ }^{2}$ \\ ${ }^{1}$ Department of Anesthesiology and Intensive Care Medicine, Tübingen University Hospital, Tübingen, Germany; \\ ${ }^{2}$ Center for Experimental Therapeutics, Brigham and Women's Hospital, Harvard Medical School, \\ Boston, Massachusetts, USA
}

Received 14 December 2004; accepted in revised form 23 March 2005

Key words: adenosine, adenosine monophosphate, adenosine triphosphate, CD39, CD73, ectonucleotidase, endothelium, hypoxia, inflammation, inosine, neutrophil, TEM, transendothelial migration

\begin{abstract}
During episodes of hypoxia and inflammation, polymorphonuclear leukocytes (PMN) move into underlying tissues by initially passing between endothelial cells that line the inner surface of blood vessels (transendothelial migration, TEM). TEM creates the potential for disturbances in vascular barrier and concomitant loss of extravascular fluid and resultant edema. Recent studies have demonstrated a crucial role for nucleotide metabolism and nucleoside signaling during inflammation. These studies have implicated multiple adenine nucleotides as endogenous tissue protective mechanisms in vivo. Here, we review the functional components of vascular barrier, identify strategies for increasing nucleotide generation and nucleoside signaling, and discuss potential therapeutic targets to regulate the vascular barrier during inflammation.
\end{abstract}

Abbreviations: AMP $-5^{\prime}$-adenosine monophosphate; APCP $-5^{\prime}$-alpha, beta-methylenediphosphate; ATP - 5-adenosine triphosphate; BMK1 - big mitogen-activated protein kinase-1; cAMP - cyclic adenosine monophosphate; CD39 - ectoapyrase; CD73 - ecto-5'-nucleotidase; ERK5 - extracellular signal-regulated kinase 5; HBP - heparin binding protein; HIF hypoxia inducible factor; ICAM - intercellular adhesion molecule; MAPK - mitogen activated protein kinase; mGluR metabotropic glutamate receptor; PMN-polymorphonuclear leukocyte (neutrophil); TEM-transendothelial migration; VASP - vasodilator-stimulated phosphoprotein

\section{Introduction}

Approximately 70 million PMN exit the vasculature per minute [1]. These inflammatory cells move into underlying tissue by initially passing between endothelial cells that line the inner surface of blood vessels. This process, referred to as transendothelial migration (TEM), is particularly prevalent in inflamed tissues, but also occurs as a natural process of leukocyte mobilization (e.g., bone marrow extravasation). Understanding the biochemical details of leukocyte-endothelial interactions is currently an area of concentrated investigation, and recent studies using genetically modified animals have identified specific molecules which function as 'bottlenecks' in the control of the inflammatory response [2]. For example, detailed studies have revealed that the process of leukocyte TEM entails a

Correspondence to: Dr Sean P. Colgan, PhD, Center for Experimental Therapeutics, Brigham and Women's Hospital, Harvard Medical School, Thorn Building 704, 75 Francis Street, Boston, MA 02115, USA. Tel: +1617-2780599; Fax: +1-617-2786957; E-mail: colgan@zeus.bwh.harvard. edu concerted series of events involving intimate interactions of a series of leukocyte and endothelial glycoproteins that include selectins, integrins, and members of the immunoglobulin supergene family [3-5]. Histologic studies of TEM reveal that PMN initially adhere to endothelium, move to nearby inter-endothelial junctions via diapedesis, and insert pseudopodia into the inter-endothelial paracellular space [6].

Successful TEM is accomplished by temporary PMN selfdeformation with localized widening of the inter-endothelial junction. Particularly during episodes of inflammation, TEM has the potential to disturb vascular barrier function and give rise to extravascular fluid extravasation and edema. However, several innate mechanisms have been described to dampen fluid loss during PMN-endothelial interactions [7]. For example, following TEM, adjacent endothelial cells appear to 'reseal,' leaving no residual inter-endothelial gaps [6]. These histologic studies are consistent with the observation that leukocyte TEM may result in little or no change in endothelial permeability to macromolecules [8-12]. In the absence of this tight and dynamic control of endothelial morphology and permeability, inter-endothelial 
gap formation during leukocyte TEM could lead to marked increases in endothelial permeability (Figure 1). However, only limited information exists regarding the biochemical events which maintain and dynamically regulate endothelial permeability in the setting of either PMN activation or TEM $[5,6]$. Current studies reveal that activated PMN release soluble factor(s) which support maintenance of endothelial permeability during PMN-endothelial interactions [7, 13, 14]. Several crosstalk pathways have been identified to protect endothelial permeability during inflammation and hypoxia and to dampen excessive fluid loss into the interstitium. Such innate protective pathways share the common strategy to increase intravascular adenosine concentrations and adenosine signaling within the inflamed or hypoxic vasculature [7, 14-18].

\section{Structural and functional elements of the vascular barrier}

The predominant barrier $(\sim 90 \%)$ to movement of macromolecules across a blood vessel wall is presented by the endothelium $[19,20]$. Passage of macromolecules across a cellular monolayer can occur via either a paracellular route (i.e., between cells) or a transcellular route (i.e., through cells). In non-pathologic endothelium, macromolecules such as albumin (molecular weight $\sim 65 \mathrm{kD}$ ) appear to cross the cell monolayer by passing between adjacent endothelial cells (i.e., paracellular) although some degree of transcellular passage may also occur $[21,22]$. Endothelial permeability is determined by cytoskeletal mechanisms that regulate lateral membrane intercellular junctions [23, 24]. Tight junctions, also known as zona occludens, comprise one type of intercellular junction. Transmembrane proteins found within this region which function to regulate paracellular passage of macromolecules include the proteins occludin, and members of the junctional adhesion molecule (JAM) and claudin families of proteins [18]. Tight junctions form narrow, cell-to-cell contacts with adjacent cells and comprise the predominant barrier to transit of macromolecules between adjacent endothelial cells [25]. Endothelial macromolecular permeability is inversely related to macromolecule size. Permeability is also dependent on the tissue of origin. For example, endothelial cells in the cerebral circulation (i.e., blood-brain barrier) demonstrate an exceptionally low permeability [26, 27]. Endothelial permeability may increase markedly upon exposure to a variety of inflammatory compounds (e.g., histamine, thrombin, reactive oxygen species, leukotrienes, bacterial endotoxins) or adverse conditions (e.g., hypoxia, ischemia) $[6,19]$. Reversible increases in endothelial permeability are produced by administration of cytochalasin or other agents that disrupt cytoskeletal microfilaments $[19,28]$. Likewise, increases in endothelial permeability are accompanied by disruption of peripheral actin microfilaments and formation of gaps between adjacent endothelial cells [19, 28]. Administration of compounds that decrease endothelial permeability result in an irregular endothelial cell contour, greater convolution of cell margins, closer cell-to-cell contact, and increased surface area and cell perimeter [28]. These changes in cell morphology are accompanied by a loss of F-actin in stress fibers, 'ruffling' of dense peripheral bands of F-actin, and increase in the polymerized actin pool without significant changes in total F-actin endothelial cell content $[23,24]$. Interestingly, these changes in intracellular actin are similar to those observed during PMN transendothelial migration [29]. By comparison, thrombininduced increases in permeability result in a centralization (and peripheral loss) of F-actin. Both of these changes (permeability and F-actin distribution) are inhibited by isoproterenol [30]. Phallacidin, an F-actin-stabilizing compound, also markedly attenuates thrombin-induced increases in permeability and accompanying morphologic changes (Figure 1).

In addition to the above components of the vascular barrier, the glycocalyx may play a role in determining movements of fluid and macromolecules across the endothelium. The endothelial glycocalyx is a dynamic extracellular matrix composed of cell surface proteoglycans, glycoproteins, and adsorbed serum proteins, implicated in the regulation and modulation of capillary tube hematocrit, permeability, and hemostasis [31]. As such, increased paracellular permeability of such molecules as water, albumin and hydroxyethyl starch can be observed following experimental degradation of the functional components of the glycocalyx [32], and functional components of this glycocalyx may be dynamically regulated by endogenous mediators such as adenosine [33].

\section{Vascular barrier during inflammation}

Ongoing inflammatory responses are characterized by dramatic shifts in tissue metabolism. These changes include large fluctuations in energy supply and demand and diminished availability of oxygen [34]. Such shifts in tissue metabolism result, at least in part, from profound recruitment of inflammatory cell types, particularly myeloid cells such as neutrophils (PMN) and monocytes. The majority of inflammatory cells are recruited to, as opposed to being resident at, inflammatory lesions, and myeloid cell migration to sites of inflammation are highly dependent on hypoxia-adaptive pathways [34, 35]. Consequently, much recent attention has focused on understanding how metabolic changes (e.g., hypoxia) relate to the establishment and propagation of the inflammatory response (Figure 2).

As outlined above, many parallels exist between hypoxic and inflamed tissues [36]. For example, during episodes of hypoxia, polymorphonuclear leukocytes (PMN) are mobilized from the intravascular space to the interstitium, and such responses may contribute significantly to tissue damage during consequent reperfusion injury [13, 37, 38]. Moreover, emigration of PMN through the endo- and epithelial barrier may lead to a disruption of such tissue barriers [39-41] and such a setting creates the potential for extravascular fluid leakage and subsequent edema forma- 


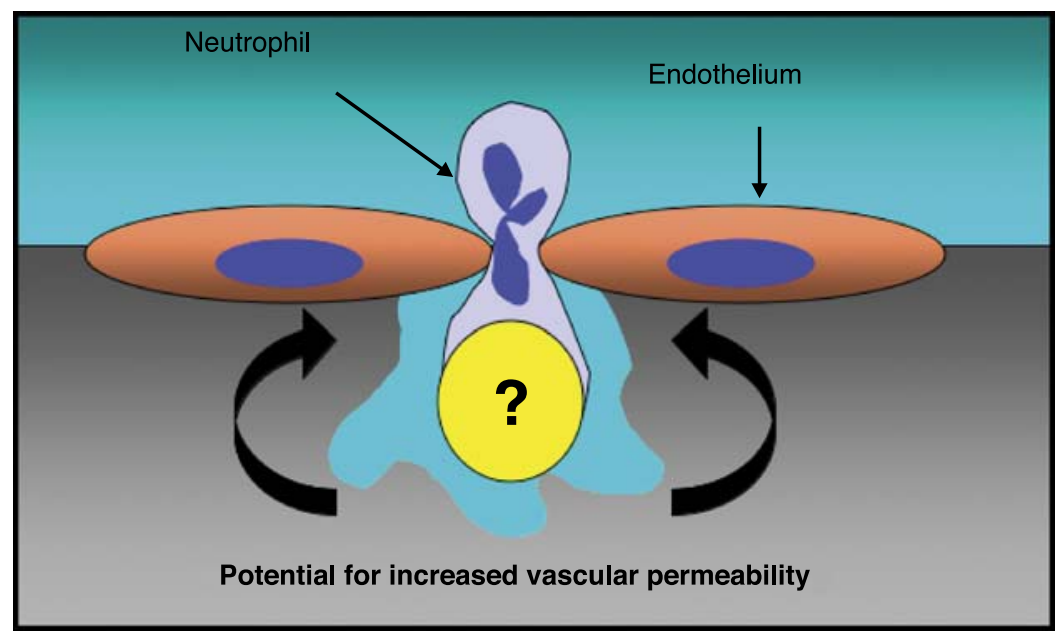

Figure 1. Neutrophil $(P M N)$ transendothelial migration $(T E M)$. PMN exit the circulation and move into underlying tissue by passing between endothelial cells. During this process, inter-endothelial gaps have the potential to increase vascular permeability, with resultant tissue edema and organ dysfunction. While the endogenous mechanism(s) which regulate these pathways remain poorly understood, work in recent years has revealed new and previously unappreciated targets for potential therapeutic intervention.

tion $[42,43]$. In contrast, transcriptional pathways mediated by hypoxia-inducible factor (HIF) may serve as a barrierprotective element during inflammatory hypoxia. For example, experimental studies of murine inflammatory bowel diseases have revealed extensive mucosal hypoxia and concomitant HIF-1 activation during colitis [44]. Mice engineered to express decreased intestinal epithelial HIF1 exhibit more severe clinical symptoms of colitis, while increased HIF levels were protective in these parameters. Furthermore, colons with constitutive activation of HIF displayed increased expression levels of HIF-regulated barrierprotective genes (multidrug resistance gene-1, intestinal trefoil factor, CD73), resulting in attenuated loss of barrier during colitis in vivo. Such studies identify HIF as a critical factor for barrier protection during mucosal inflammation and hypoxia [44].

\section{Barrier disruptive pathways}

Macromolecule transit across blood vessels has evolved to be tightly controlled. Relatively low macromolecular permeability of blood vessels is essential for maintenance of a physiologically optimal equilibrium between intravascular and extravascular compartments [45, 46]. Endothelial cells are primary targets for leukocytes during episodes of infection, ischemic or traumatic injury, which all together can result in an altered barrier function. Disturbance of endothelial barrier during these disease states can lead to deleterious loss of fluids and plasma protein into the extravascular compartment. Such disturbances in endothelial barrier function are prominent in disorders such as shock and ischemia-reperfusion and contribute significantly to organ dysfunction [47-51].

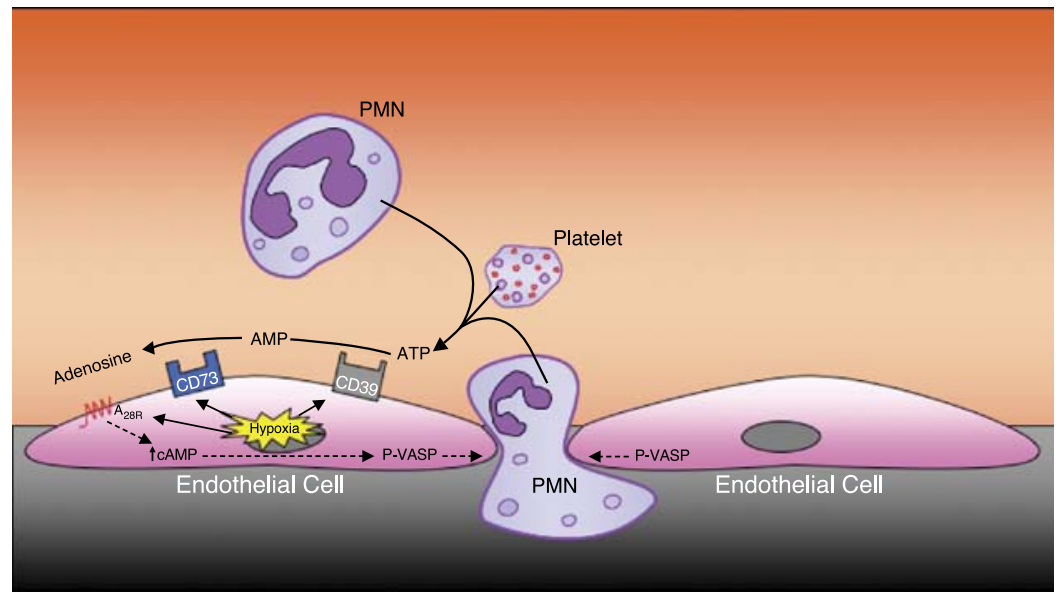

Figure 2. Proposed model of coordinated nucleotide metabolism and nucleoside signaling in posthypoxic endothelial cells. In areas of ongoing inflammation, diminished oxygen supply coordinates the induction of CD39, CD73, and AdoRA $2 \mathrm{~B}$. At such sites, activated PMN provide a readily available extracellular source of ATP, that through two enzymatic steps results in the liberation of extracellular adenosine. Activation of surface AdoR functions to promote the re-establishment of vascular integrity. Under such circumstances, PMN appear to "close the door after exiting." 
Previous studies have indicated that activated PMN release a number of soluble mediators, which dynamically influence vascular permeability during transmigration. As such, PMN have been shown to liberate factors that can either disrupt or protect the endothelial barrier: For example, it was recently shown that activation of PMN through $\beta 2$ integrins elicits the release of soluble factor(s) which induce endothelial cytoskeletal rearrangement, gap formation and increased permeability [52]. This PMN-derived permeabilizing factor was subsequently identified as heparin-binding protein (HBP), also called azurocidin or CAP37 [52], a member of the serprocidin family of cationic peptides [53]. HBP, but not other neutrophil granule proteins (e.g., elastase, cathepsin G), was shown to induce $\mathrm{Ca}^{2+}$-dependent cytoskeletal changes in cultured endothelia and to trigger macromolecular leakage in vivo. Interestingly, HBP regulation of barrier may not be selective for PMN, and in fact, endothelial cells themselves are now a reported source of HPB [54]. It is therefore possible that endothelia may self-regulate permeability through HBP under some conditions, and that mediators found within the inflammatory milieu may also increase endothelial permeability.

Similarly, PMNs were observed to significantly alter endothelial permeability by release of glutamate, following FMLP activation. This crosstalk pathway appears to be of particular importance for the regulation of the vascular barrier of the brain ("blood-brain barrier"). In fact, treatment of human brain endothelia with glutamate or selective, mGluR group I or III agonists resulted in a timedependent loss of phosphorylated vasodilator-stimulated phosphoprotein (VASP) and significantly increased endothelial permeability. Glutamate-induced decreases in brain endothelial barrier function and phosphorylated VASP were significantly attenuated by pretreatment of human brain endothelia with selective mGluR antagonists. Even in an in vivo hypoxic mouse model, the pretreatment with mGluR antagonists significantly decreased fluorescein isothiocyanate-dextran flux across the blood-brain barrier, suggesting that activated human PMNs release glutamate and that endothelial expression of group I or III mGluRs function to decrease human brain endothelial VASP phosphorylation and barrier function [13].

A recently described gene regulatory pathway revealed a critical role for BMK1/ERK5 in maintaining the endothelial barrier and blood vessel integrity: A targeted deletion of big mitogen-activated protein kinase1 gene (BMK1) (also known as ERK5, member of the MAPK family), in adult mice leads to disruption of the vascular barrier. Histological analysis of these mice revealed that, after BMK1 ablation, hemorrhages occurred in multiple organs in which endothelial cells lining the blood vessels became round, irregularly aligned, and, eventually, apoptotic. In vitro removal of $\mathrm{BMK} 1$ protein also led to the death of endothelial cells partially due to the deregulation of transcriptional factor $\mathrm{MEF} 2 \mathrm{C}$, which is a direct substrate of BMK1. Additionally, endothelial-specific BMK1-deletion leads to cardiovascular defects identical to that of global BMK1-deletion. Taken together, these studies identify the BMK1 pathway as critical for endothelial function and for maintaining blood vessel integrity [55].

\section{Barrier protective pathways}

Acute increases in vascular permeability to macromolecules closely coincides with tissue injury of many etiologies, and can result in fluid loss, edema, and organ dysfunction [19, 56, 57]. Previous studies have indicated that extracellular nucleotide metabolites may function as an endogenous protective mechanism during hypoxia and ischemia [58-60]. One important factor may be increased production of endogenous adenosine, a naturally occurring anti-inflammatory agent [60-62]. Several lines of evidence support this assertion. First, adenosine receptors are widely expressed on target cell types as diverse as leukocytes, vascular endothelia, and mucosal epithelia and have been studied for their capacity to modulate inflammation [63]. Second, murine models of inflammation provide evidence for adenosine receptor signaling as a mechanism for regulating inflammatory responses in vivo. For example, mice deficient in the $\mathrm{A}_{2 \mathrm{~A}}$-adenosine receptor (AdoRA $\mathrm{A}_{2 \mathrm{~A}}$ ) show increased inflammation-associated tissue damage [64]. Third, hypoxia is a common feature of inflamed tissues [34] and is accompanied by significantly increased levels of adenosine [65-67]. At present, the exact source of adenosine is not well defined, but likely results from a combination of increased intracellular metabolism and amplified extracellular phosphohydrolysis of adenine nucleotides via surface ecto-nucleotidases.

The vascular endothelium is the primary interface between a hypoxic insult and the surrounding tissues. At the same time, the endothelium is central to the orchestration of leukocyte trafficking in response to chemotactic stimuli. This critical anatomic location places vascular endothelial cells in an ideal position to coordinate extracellular metabolic events important to endogenous antiinflammatory responses. We recently identified a neutrophil-endothelial cell crosstalk pathway that is coordinated by hypoxia. This pathway utilizes extracellular nucleotide substrates, liberated from different cell types. Extracellular ATP release has been shown from endothelial cells, particularly under sheer conditions or hypoxia. In addition, fMLP activated neutrophils can release ATP, however the exact mechanism through that these cells release ATP remains currently unknown (see below) [14, 47]. Activated platelets comprise an additional source for extracellular adenine nucleotides $[68,69]$. The role of endothelial CD39 (Ecto-apyrase, conversion of ATP/ADP to AMP) has been viewed as a protective, thromboregulatory mechanism for limiting the size of the hemostatic plug $[69,70]$. Metabolism of adenine nucleotides derived from activated platelets is crucial in limiting excessive platelet aggregation and thrombus formation [71, 72]. Similarly, excessive platelet accumulation and recruitment can be treated with the use of soluble forms of CD39 [73, 74]. Moreover, a thromboregulatory role was demonstrated in a model of stroke, where $c d 39$-null mice showed increased infarction size that was reduced by treatment with soluble CD39 [75]. 
Surprisingly, targeted disruption of $c d 39$ resulted in prolonged bleeding and increased vascular leak and fibrin deposition in hypoxemia [76], suggesting a dual role for ATP metabolism by CD39 in modulating hemostasis and thrombotic reactions. Moreover, this observation may be related to an activation and desensitization of the purinergic type $\mathrm{P}_{2} \mathrm{Y}_{1}$ receptor. Activation of the $\mathrm{P}_{2} \mathrm{Y}_{1}$-platelet receptor appears to be crucial in the activation process of platelets. As such, $\mathrm{P}_{2} \mathrm{Y}_{1}$ deficient mice exhibit signs of prolonged bleeding time and resistance to thromboembolism [77]. In contrast to these studies, we observed a barrier-protective influence during hypoxia that was not related to the activation of PX receptors, but to a downstream metabolism and signaling of ATP metabolites (esp. adenosine).

Extracellular ATP is readily converted on the endothelial surface to adenosine, due to the enzymatic function of CD39 and CD73 (5'-ecto-nucleotidase, conversion of AMP to adenosine). Such adenosine binds to surface expressed PMN adenosine receptors to limit excessive accumulation of PMN within tissues, and as such, functions as a feedback loop to attenuate potential tissue injury [14]. With regard to this latter point, it was recently shown that hypoxia coordinates both, transcriptional and metabolic control of the surface ecto-nucleotidases CD39 and CD73 $[14,15,78]$, and as such, significantly amplifies the extracellular production of adenosine from adenine nucleotide precursors. In fact when using cd39- and cd73-null animals we found that extracellular adenosine, produced through adenine nucleotide metabolism during hypoxia, is a potent anti-inflammatory signal for PMN in vitro and in vivo. These findings identify CD39 and CD73 as critical control points for endogenous adenosine generation and implicate this pathway as an innate mechanism to attenuate excessive tissue PMN accumulation [14].

In addition to a role in limiting excessive neutrophil tissue accumulation, $\mathrm{CD} 39$ and $\mathrm{CD} 73$ are also critical control points for vascular permeability. For the purpose of investigating overall organ vascular permeability, we used Evan's blue dye, which tightly binds to plasma albumin [79]. To do this, mice were administered $0.2 \%$ Evan's blue dye $(0.5 \%$ in PBS) by intravenous tail vein injection, subjected to room air (normoxia) or normobaric hypoxia $\left(8 \% \mathrm{O}_{2} / 92 \% \mathrm{~N}_{2}\right)$. At the end of exposure, animals were anesthetized, heparinized (50 U by i.p. injection) and fluid overloaded (3 cc normal Ringer's solution i.p.). Animals were then exsanguinated by femoral cut-down to flush all vascular beds, and tissues were harvested. Tissues were rinsed in PBS, and Evan's blue was extracted with formamide at $56{ }^{\circ} \mathrm{C}$ for $2 \mathrm{~h}$, and quantified at $610 \mathrm{~nm}$ with subtraction of reference absorbance at $450 \mathrm{~nm}$. This model entails the quantification of formamide-extractable Evans blue [80] from tissues of mice as a readout for overall vascular permeability of different organs. In fact we found that vascular permeability in tissues derived from animals subjected to normobaric hypoxia $\left(8 \% \mathrm{O}_{2} / 92 \% \mathrm{~N}_{2}\right)$ ranged from two to four-fold more permeant to Evans blue than normoxic controls [14].

In order to identify the role of CD73 in vascular permeability, we used this model in wild-type or $c d 73^{-1-}$ mice administered either vehicle or the CD73 inhibitor $\alpha, \beta$-methylene ADP (APCP). These studies revealed a profound increase of hypoxia-elicited dysfunction of the vascular barrier in different organs (lung, heart, intestine, kidneys) following CD73 inhibition or deletion. Vascular leak associated with hypoxia was, at least in part, reversed by reconstitution with soluble $5^{\prime}$-nucleotidase and adenosine receptor agonists in the cd73-null mice. Histological examination of lungs from hypoxic $c d 73^{-1-}$ revealed perivascular interstitial edema associated with inflammatory infiltrates surrounding larger pulmonary vessels [81]. Taken together, these studies identify CD73 as a critical mediator of vascular permeability in vivo. When measuring vascular permeability during hypoxia in mice with targeted deletion of CD39, similar increases in vascular barrier function could be observed in different organs [14]. Taken together, these studies have identified adenosine generation of the hypoxic vasculature via nucleotide-phosphohydrolysis as a critical cellular strategy to generate adenosine and maintain vascular barrier function.

Influence of adenosine receptor activation on endothelial barrier function

In vitro studies of endothelial permeability suggested, that activation of a specific endothelial adenosine receptor, the AdoRA $_{2 \mathrm{~B}}$, leads to a barrier resealing response following PMN transmigration [7]. Thus we were able to show that of the four different adenosine receptors that are expressed by endothelia, only the $\mathrm{AdoR}_{2 \mathrm{~B}}$ is selectively induced by hypoxia [14]. Activation of the $\operatorname{AdoRA}_{2 \mathrm{~B}}$ is associated with increases in intracellular cAMP concentration due to the activation of the adenylate cyclase [60]. By inhibition of cAMP formation, the resealing of the endothelial barrier during PMN transmigration can be obviated [7]. Such increases in cAMP following activation of the $\mathrm{AdoRA}_{2 \mathrm{~B}}$ lead to an activation of protein kinase A (PKA) [18]. Further studies revealed a central role of PKA-induced phosphorylation of vasodilator-stimulated phosphoprotein (VASP), a protein responsible for controlling the geometry of actin-filaments [82]. Adenosine-receptor mediated phosphorylation of VASP is responsible for changes in the geometry and distribution of junctional proteins, thereby affecting the characteristics of the junctional complex and promoting increases in barrier function $[18,83]$.

\section{Inflammatory hypoxia}

\section{Hypoxia and vascular barrier function}

A number of studies have identified perturbations of endothelial permeability associated with hypoxia $[84,85]$. In addition, tissue hypoxia has been implicated as a contributing factor to inflammatory diseases initiated at the vascular surface. For example, ongoing inflammatory responses are characterized by dramatic shifts in tissue metabolism, including lactate accumulation, increased 
nucleotide metabolism, and diminished availability of oxygen [86-88]. However, the exact mechanism by which hypoxia causes changes in vascular permeability remains the center of debate. In addition to the observation that hypoxia causes a disruption of the vascular barrier, several studies were able to identify hypoxia-elicited crosstalk pathways, that are involved in maintaining the vascular barrier and limiting excessive inflammatory responses. As outlined above, targeted disruption of such pathways is associated with dramatic increases in vascular permeability during hypoxia exposure and increases in myeloid cell accumulation within the hypoxic tissues [14, 15, 47, 81].

\section{Increases in inflammatory cell accumulation during hypoxia}

During episodes of hypoxia or during ischemia-reperfusion, the PMN influx across the protective endothelium creates the potential of endothelial barrier dysfunction, loss of fluid and edema formation [40, 89]. The myeloid cell migration to sites of inflammation are highly dependent on hypoxia adaptive pathways [34] and HIF-1 $\alpha$ plays a critical role in this process. This transcription factor consists of two subunits HIF-1 $\alpha$ and HIF-1 $\beta$. Under conditions such as normoxia, the HIF-1 $\alpha$ subunit undergoes mechanisms of enzymatic degradation. In contrast to its degradation during non-hypoxic states, degradation of the $\alpha$ subunit is inhibited under hypoxic conditions, leading to rapid accumulation of the protein. HIF- $1 \alpha$ and HIF-1 $\beta$ dimerize to the active protein that translocates to the nucleus and binds to an hypoxia response element within the promoter region of target genes, thereby altering the transcription and translation of specific hypoxia elicited gene products [90].

Moreover, previous studies demonstrate, that HIF- $1 \alpha$ is essential for myeloid cell-mediated inflammation. As outlined above, metabolic shifts during inflammation can result in significant tissue hypoxia, with resultant induction of hypoxia-responsive genes. As such, recent studies show that leukocyte functional responses are influenced by hypoxia on a transcriptional level. Increases of leukocyte accumulation within hypoxic tissues are transcriptiondependent and can be specifically blocked by antibodies directed against $\beta 2$-integrins. In addition, analysis of the $\beta 2$-integrin mRNA and protein shows a dramatic increase with hypoxia. Subsequent studies identified a binding site for hypoxia-inducible factor 1 (HIF-1) in the CD18 gene. This gene encodes the subunit common to all four known types of $\beta 2$-integrin heterodimer. HIF-1 binding was demonstrated in vivo, and mutational analysis of the HIF1 site within the CD18 promoter resulted in a loss of hypoxia inducibility, suggesting that hypoxia induces leukocyte $\beta 2$-integrin expression and function by transcriptional mechanisms dependent upon HIF-1 [35]. Moreover, myeloid cellular ATP-pools are significantly reduced in the absence of HIF-1 $\alpha$, leading to profound impairment of myeloid cell aggregation, motility, invasiveness and bacterial killing [34]. Shifts in tissue metabolism result, at least in part, from extensive recruitment of inflammatory cells, particularly myeloid cells such as neutrophils (PMN) and monocytes [36]. However, the direct relationship between PMN activation and increased endothelial permeability is not clear. While PMN accumulation and increased vascular permeability are often coincidental, particularly at sites of inflammation/hypoxia, PMN activation can occur with limited or no net changes in endothelial permeability [8-10, 12]. Moreover, while PMN depletion has been demonstrated to decrease organ injury in some models of ischemia and reperfusion [91], PMN have been demonstrated to exert a protective effect in other models. Therefore, further information regarding PMN-endothelial cell interactions and their influence on endothelial permeability may provide a better understanding of the regulation of endothelial permeability.

\section{Purine signaling during inflammation and hypoxia}

\section{PMN release nucleotides}

As stated earlier, the transendothelial migration of PMN during episodes of inflammation and hypoxia can be associated with fluid accumulation, edema and organ dysfunction as a cause of increased vascular permeability. During TEM, neutrophils primarily interact with endothelial cells and it is well documented that transmigration of neutrophils may lead to an alteration of barrier function. However their interactions with the endothelium do not inevitably result in endothelial barrier dysfunction: there is also evidence that PMN do not affect vascular permeability despite extensive non-inflammatory PMN-endothelial adhesion and subsequent transendothelial PMN migration. Although many studies indicate that activated PMN lead rather to disruption of the endothelial barrier, an increase of endothelial barrier permeability upon exposure to activated PMN has also been demonstrated [7, 14]. These studies show that PMN derived soluble mediators like adenosine and AMP are capable to increase the endothelial barrier function via activation of the AdoRA $_{2 B}$ adenosinereceptor [59]. In addition, we showed that neutrophils also release ATP following activation, and such ATP is coordinately hydrolyzed to adenosine at the endothelial cell surface particularly under hypoxic conditions by hypoxiainduced CD39 and CD73 [14]. Several mechanisms for ATP release have been proposed, including direct transport through ATP-binding cassette (ABC) proteins, transport through connexin hemichannels, as well as vesicular release [92]. At present, the mechanism by which leukocytes release ATP remains unclear and is the subject of ongoing studies. Moreover, studies directed at understanding extracellular metabolism of nucleotides in cell and tissue responses now suggest that a number of different cells can release ATP in an active manner [93]. In addition to PMN, also activated platelets comprise an important source for extracellular adenine nucleotides [68, 69]. However, their relative contribution to regulating the vascular barrier or inflammation is currently not clear. 
Nucleotide phosphohydrolysis is increased during hypoxia

The major pathway for extracellular hydrolysis of ATP and ADP is the ecto-nucleoside triphosphate diphosphohydrolase (NTPDase) [94], previously identified as ecto-ATPase, ecto-ATPDase or CD39 [95, 96]. In addition to modulating extracellular nucleoside generation, CD39 has been considered in the modulation of platelet purinoreceptor activity by the sequential hydrolysis of extracellular ATP or ADP to AMP $[96,97]$. This thromboregulatory potential of CD39 has been demonstrated by the generation of mutant mice with disruption of the CD39 gene [76], and by a series of experiments where high levels ATPDase expression are attained by adenoviral vectors in the injured vasculature [98-100].

Ecto-5'-nucleotidase (CD73) is a membrane bound glycoprotein which functions to convert the adenine nucleotide AMP into adenosine, which in turn can activate transmembrane adenosine receptors or can be internalized through dipyridamole-sensitive carriers [60]. These pathways have been shown to result in such diverse endpoints as regulation of endothelial barrier [7], stimulation of epithelial electrogenic chloride secretion (responsible for mucosal hydration) [101], and promotion of lymphocyte- endothelial adhesion [102]. Targeted over expression of lymphocyte CD73 has recently revealed that increased adenosine resulting from enhanced CD73 expression could contribute to thymocyte apoptosis in adenosine deaminase deficiency [103]. Endothelial cells of many origins express constitutive CD73. The primary function attributed to endothelial CD73 has been catabolism of extracellular nucleotides, although CD73 may also mediate lymphocyte binding under some circumstances [102].

As outlined above, multiple cell types release adenine nucleotides under conditions of limited oxygen availability. Extracellular nucleotides are metabolized to adenosine by CD39 and CD73 and subsequent activation of surface adenosine receptors has been shown to regulate endothelial and epithelial barrier function [7]. Microarray RNA analysis revealed an increase in CD73 and CD39 in hypoxic endothelial and epithelial cells $[14,15]$. Metabolic studies of CD39/CD73 function revealed that hypoxia enhances CD39/CD73 function as much as six-fold over normoxia. Examination of the $\mathrm{CD} 73$ gene promoter identified a binding site for hypoxia-inducible factor-1 (HIF-1) and inhibition of HIF-1alpha expression by antisense oligonucleotides resulted in significant inhibition of hypoxia-inducible CD73 expression. Studies using luciferase reporter constructs showed a significant increase in activity in cells subjected to hypoxia, which was lost in truncated constructs lacking the HIF-1 site. Mutagenesis of the HIF-1alpha binding site resulted in a nearly complete loss of hypoxiainducibility. In vivo studies in a murine hypoxia model revealed that hypoxia-induced CD73 may serve to protect the epithelial [15] and endothelial [81] barrier, since the CD73 inhibitor alpha,beta-methylene ADP (APCP) promotes increased intestinal permeability and $\mathrm{cd} 73^{-1-}$ mice show increased vascular permeability during hypoxia exposure. These results identify a HIF-1-dependent regu- latory pathway for $\mathrm{CD} 73$ and indicate the likelihood that CD39/CD73 protects the epithelial and vascular barrier during hypoxia $[14,15,81]$.

\section{Adenosine concentrations in hypoxia}

Extracellular adenosine has been widely implicated in adaptive responses to hypoxia. Thus, it is well documented that adenosine tissue and plasma levels are increased during hypoxia, however, mechanisms of this response have yet to be further elucidated [66, 104]. For example, in human volunteers exposed to ambient hypoxia $\left(\mathrm{SpO}_{2}=\right.$ $80 \%$ over $20 \mathrm{~min}$ ), plasma adenosine concentrations increased from 21 to $51 \mathrm{nM}$ in the presence of dipyridamole, an inhibitor of adenosine reuptake [105]. Similarly, when measuring adenine nucleotide concentrations in neurally and vascularly isolated, perfused skeletal muscles of anesthetized dogs, normobaric hypoxia is associated with increases of adenosine in the venous blood, but not of AMP, ADP or ATP [106]. A possible role for adenosine during hypoxia may include vasodilatory characteristics. For instance, it has been suggested that elevations in intracellular nitric oxide as a result of AdoRA $\mathrm{A}_{1}$ activation may be responsible for the vasodilatory properties of adenosine [107-109]. Due to its vasodilator properties, increases in adenosine tissue concentrations during hypoxia may promote blood flow to hypoxic tissues, thereby providing an innate adaptive pathway to hypoxia. In addition, there is evidence that hypoxia-induced vascular leak is, at least in part, controlled by adenosine. In vitro and in vivo studies indicated that endogenously generated adenosine, through activation of PMN adenosine AdoRA $\mathrm{A}_{2 \mathrm{~A} / 2 \mathrm{~B}}$ receptors, functions as an anti-adhesive signal for PMN binding to microvascular endothelia $[47,64,110]$. Other studies revealed that adenosine derived via nucleotide phosphohydrolysis is critical in eliciting decreases in endothelial paracellular permeability [7] and that such responses are dramatically enhanced by hypoxia $[14,15]$. In addition, some studies have suggested that uptake of adenosine by equilibrative nucleoside transporters (ENTs) in cultured myocardial cells are decreased during hypoxia. This might resemble an additional cellular strategy to increase extracellular levels of adenosine during hypoxia [111]. However, this has not yet been investigated within the endothelium or the vasculature, and the physiological relevance of this adaptive pathway currently remains unclear.

\section{Increased adenosine signaling by hypoxia}

Several studies had demonstrated a functional role for hypoxia-regulated CD39 and CD73 in promoting endothelial permeability [14, 15, 47], however, the role of signal transduction via endothelial adenosine receptors in the post-hypoxic vasculature remained unclear until recently. In order to identify changes in endpoint signaling (i.e., adenosine receptor activation) in the post-hypoxic endothelium, we profiled the relative expression of adenosine receptors in normoxic and hypoxic endothelial cells. Interestingly, these experiments demonstrated that the 
AdoRA $_{2 B}$ was selectively induced by hypoxia, and that all other isoforms were either not changed $\left(\mathrm{AdoRA}_{3}\right)$ or significantly down-regulated (AdoRA $A_{1}$ and AdoRA $_{2 A}$ ) [14]. Such microarray results were verified by real-time PCR and measurement of surface protein levels and consistently confirmed selective induction of the AdoRA $A_{2 B}$ receptor by hypoxia. To determine whether such hypoxiainduced AdoRA $_{2 \mathrm{~B}}$ expression was functional, endothelial permeability assays were employed using the selective AdoRA $_{2 \mathrm{~B}}$ antagonist MRS 1754 [112] and demonstrated a significantly shift in the adenosine dose-response curve suggesting that AdoR $\mathrm{A}_{2 \mathrm{~B}}$ responses are amplified in posthypoxic endothelia. However, further studies in vivo to verify these in vitro findings in genetically engineered animals using targeted deletion of individual adenosine receptors have yet to be performed to follow up on these observations.

\section{Nucleotide and inosine signaling}

Purine signaling is presently a field of intense investigation. Adenosine as an extracellular nucleoside interacts via P1 receptors. Four P1 receptor subtypes have been identified until now, namely $\operatorname{AdoRA}_{1}, \operatorname{AdoRA}_{2 \mathrm{~A}}, \operatorname{AdoRA}_{2 \mathrm{~B}}$ and AdoRA $_{3}$ and the growth hormone secretagogue receptor GHSR [60]. Extracellular nucleotides as signaling molecules interact through to different categories of P2-types of cell surface receptors: P2Y type G protein coupled receptors, and $\mathrm{P} 2 \mathrm{X}$ type ligand gated ion channels. Eight subtypes of $\mathrm{P} 2 \mathrm{Y}$ receptors have been characterized at present, which are either activated by the adenine nucleotides ATP or ADP or respectively by the uridine nucleotides UTP or UDP. One P2Y subtype is activated by both, adenine and uridine nucleotides [113-116]. However, there can be large differences between different species (e.g., between human and rat) with regard to $\mathrm{P} 2$ receptor selectivity for adenine or uridine nucleotides [117]. Despite of the fact that the vascular endothelium and multiple cell types of the immune system (including PMN) express different P2 receptors [113, 118], only very little is known about the effect of nucleotide signaling on neutrophilendothelial interaction. In fact, the cell physiology of these receptors and the precise function of the multiple P2 receptor subtypes remains to be understood. As currently selective blockers of these receptors are being synthesized, we can soon hope for a more precise understanding of how nucleotide signaling modulates immune function [119].

There is no unequivocal evidence that AMP can function as a signaling molecule. Recently, Inbe et al. suggested that the orphan receptor GPR80/99 may function as an AMP receptor [120]. In contrast, there is now convincing evidence that GPR80/99 binds $\alpha$-ketoglutarate, thereby functioning as receptor for citric acid cycle intermediates [121]. In addition, other studies demonstrated that GPR80/99 does not bind adenosine or AMP [122]. Therefore, there is now consensus that GPR80/99 is not a nucleotide receptor [123].

In contrast to adenosine, which has for long been considered an important modulator of immune function and inflammation, its major degradation product inosine was originally thought to have no biological effects. However, some recent studies have suggested that inosine itself may influence immune function and tissue protection [61]. Similar to adenosine, inosine concentrations are increased during hypoxia and during ischemia/reperfusion [124]. As such, inosine was found to bind to the adenosine AdoRA $_{3}$ receptor subtype and cause mast cell degranulation [125] or cutaneous vasodilatation [126]. In addition, inosine was found to be involved in the down-regulation of tissue damage under different inflammatory conditions in vivo via activation of $\mathrm{AdoRA}_{2 \mathrm{~A}}$ and $\mathrm{AdoRA}_{3}$ adenosine receptors [127]. However, specific inosine receptors have not yet been described. In addition, it remains unclear whether endogenously produced inosine is sufficient to exert tissue protective influences in vivo, particularly since the AdoRA $A_{3}$ receptors show only a low affinity for inosine. However, interstitial concentrations of inosine may be high enough to activate $\mathrm{AdoRA}_{3}$ receptors during pathophysiological states such as tissue hypoxia [125]. In addition, it is possible that inosine signaling may contribute to the effects of adenosine signaling, since exogenous administration of large doses of inosine were found to decrease ischemia reperfusion injury in several tissues $[61,125,128]$. Taken together, the overall contribution of inosine signaling to the regulation of the vascular function remains to be seen.

\section{Potential therapeutic developments}

Several inflammatory diseases (e.g., sepsis, acute respiratory failure, colitis) are characterized by vascular barrier dysfunction, leading to fluid extravasation and edema formation [129-131]. As outlined above, endothelial nucleoside signaling creates the potential for therapeutically modulating the vascular barrier function. For example, promoting vascular adenosine signaling by increasing extracellular adenosine generation, activation of specific vascular adenosine receptors, or impeding with adenosine breakdown may potentially dampen vascular barrier impairment during hypoxia and inflammation. Several experimental studies have examined this approach. Cohen et al. investigated the influence of the adenosine deaminase inhibitor $2^{\prime}$-deoxy-coformycin (pentostatin) in an animal model of sepsis (cecal ligation and puncture model) [132]. Using intravital microscopy, the authors demonstrated that septic mice treated with pentostatin revealed decreased leukocyte rolling and adhesion. In addition, circulating levels of interleukin- 6 and tumor-necrosis-factor- $\alpha$ were decreased, and survival was increased after $48 \mathrm{~h}$. At the same time, leakage of fluorescein-isothiocyanatelabeled albumin was decreased following pentostatin treatment, suggesting that inhibition of adenosine breakdown during sepsis is associated with improved vascular barrier function and decreased inflammatory activation. An alternate strategy to promote vascular adenosine signaling may include administration of soluble nucleotidase. As outlined above, nucleotidases convert AMP to adenosine, thereby increasing adenosine levels. Using this principle, it was recently shown that administration of 5-nucleotidase to 
mice during hypoxia significantly diminished vascular leak syndrome [81]. Moreover, we recently demonstrated that administration of the specific AdoRA $_{2 \mathrm{~B}}$ antagonist MRS 1754 is associated with decreased endothelial adenosine responses, causing increased leakage of $70 \mathrm{kD}$ dextran molecules [14]. These studies were confirmed using an in vivo model of pulmonary barrier function, showing increased albumin leakage after hypoxia exposure in mice treated with MRS 1754 [81]. These experiments suggest that therapeutic use of a specific agonist of the AdoRA $A_{2 B}$ may dampen edema and fluid extravasation in patients suffering from inflammatory vascular leak syndrome(s). Despite of the fact that our understanding of neutrophilendothelial interaction during inflammation and hypoxia has considerably advanced over the last years, and purine metabolism and signaling clearly offers novel therapeutic targets in the treatment of inflammation, these experimentally derived ideas have yet to be fully integrated into clinical practice.

\section{References}

1. Dancey JT, Deubelbeiss KA, Harker LA, Finch CA. Neutrophil kinetics in man. J Clin Invest 1976; 58: 705-15.

2. Ley K. Pathways and bottlenecks in the web of inflammatory adhesion molecules and chemoattractants. Immunol Res 2001; 24: 87-95.

3. Shimizu Y, Rose DM, Ginsberg MH. Integrins in the immune system. Adv Immunol 1999; 72: 325-80.

4. Gonzalez-Amaro R, Sanchez-Madrid F. Cell adhesion molecules: Selectins and integrins. Crit Rev Immunol 1999; 19: 389-429.

5. Ley K. Integration of inflammatory signals by rolling neutrophils. Immunol Rev 2002; 186: 8-18.

6. Dejana E, Spagnuolo R, Bazzoni G. Interendothelial junctions and their role in the control of angiogenesis, vascular permeability and leukocyte transmigration. Thromb Haemost 2001; 86: 308-15.

7. Lennon PF, Taylor CT, Stahl GL, Colgan SP. Neutrophil-derived 5 '-adenosine monophosphate promotes endothelial barrier function via CD73-mediated conversion to adenosine and endothelial $A_{2 B}$ receptor activation. J Exp Med 1998; 188: 1433-43.

8. Dejana E, Corada M, Lampugnani MG. Endothelial cell-to-cell junctions. FASEB J 1995; 9: 910-8.

9. Lewis RE, Granger HJ. Diapedesis and the permeability of venous microvessels to protein macromolecules: The impact of leukotriene $\mathrm{B}_{4}\left(\mathrm{LTB}_{4}\right)$. Microsc Res 1988; 35: 27-47.

10. Meyrick B, Hoffman LH, Brigham KL. Chemotaxis of granulocytes across bovine pulmonary artery intimal explants without endothelial cell injury. Tissue Cell 1984; 16: 1-16.

11. Shaw JO, Henson PM. Pulmonary intravascular sequestration of activated neutrophils. Am J Pathol 1982; 108: 17-23.

12. Huang A, Furie M, Nicholson S et al. Effects of human neutrophil chemotaxis across human endothelial cell monolayers on the permeability of these monolayers to ions and macromolecules. J Cell Physiol 1988; 135: 355-66.

13. Collard CD, Park KA, Montalto MC et al. Neutrophil-derived glutamate regulates vascular endothelial barrier function. J Biol Chem 2002; 2002: 14801-11.

14. Eltzschig HK, Ibla JC, Furuta GT et al. Coordinated adenine nucleotide phosphohydrolysis and nucleoside signaling in posthypoxic endothelium: Role of ectonucleotidases and adenosine $\mathrm{A}_{2 \mathrm{~B}}$ receptors. J Exp Med 2003; 198: 783-96.

15. Synnestvedt K, Furuta GT, Comerford KM et al. Ecto-5'-nucleotidase (CD73) regulation by hypoxia-inducible factor-1 (HIF-1) mediates permeability changes in intestinal epithelia. J Clin Invest 2002; 110: 993-1002.
16. Collard CD, Bukusoglu C, Agah A et al. Hypoxia-induced expression of complement receptor type 1 (CR1, CD35) in human vascular endothelial cells. Am J Physiol 1999; 276: C450-8.

17. Narravula S, Lennon PF, Mueller BU, Colgan SP. Regulation of endothelial CD73 by adenosine: Paracrine pathway for enhanced endothelial barrier function. J Immunol 2000; 165: 5262-8.

18. Comerford KM, Lawrence DW, Synnestvedt K et al. Role of vasodilator-stimulated phosphoprotein in PKA-induced changes in endothelial junctional permeability. FASEB J 2002; 16: 583-5.

19. Stevens T, Garcia JGN, Shasby DM et al. Mechanisms regulating endothelial cell barrier function. Am J Physiol Lung Cell Mol Physiol 2000; 279: L419-22.

20. Stevens T, Creighton J, Thompson WJ. Control of cAMP in lung endothelial cell phenotypes. Implications for control of barrier function. Am J Physiol 1999; 277: L119-26.

21. Stan RV. Structure and function of endothelial caveolae. Microsc Res Tech 2002; 57: 350-64.

22. Michel CC. Capillaries, caveolae, calcium and cyclic nucleotides: A new look at microvascular permeability. J Mol Cell Cardiol 1998; 30: 2541-6.

23. Worthylake RA, Burridge K. Leukocyte transendothelial migration: Orchestrating the underlying molecular machinery. Curr Opin Cell Biol 2001; 13: 569-77.

24. Schoenwaelder SM, Burridge K. Bidirectional signaling between the cytoskeleton and integrins. Curr Opin Cell Biol 1999; 11: 274-86.

25. Tsukita S, Furuse M, Itoh M. Multifunctional strands in tight junctions. Nat Rev Mol Cell Biol 2001; 2: 285-93.

26. Rubin LL. Endothelial cells: Adhesion and tight junctions. Curr Opin Cell Biol 1992; 4: 830-3.

27. Janzer RC, Raff MC. Astrocytes induce blood-brain barrier properties in endothelial cells. Nature 1987; 325: 253-7.

28. Moore TM, Chetham PM, Kelly JJ, Stevens T. Signal transduction and regulation of lung endothelial cell permeability. Interaction between calcium and cAMP. Am J Physiol 1998; 275: L203-22.

29. Burns AR, Bowden RA, MacDonell SD et al. Analysis of tight junctions during neutrophil transendothelial migration. J Cell Sci 2000; 113: 45-57.

30. Minnear FL, DeMichele MAA, Moon DG et al. Isoproterenol reduces thrombin-induced pulmonary endothelial permeability in vitro. Am J Physiol 1989; 257: H1613-23.

31. Weinbaum S, Zhang X, Han Y et al. Mechanotransduction and flow across the endothelial glycocalyx. Proc Natl Acad Sci USA 2003; 100: 7988-95.

32. Rehm M, Zahler S, Lotsch M et al. Endothelial glycocalyx as an additional barrier determining extravasation of $6 \%$ hydroxyethyl starch or $5 \%$ albumin solutions in the coronary vascular bed. Anesthesiology 2004; 100: 1211-23.

33. Platts SH, Duling BR. Adenosine A3 receptor activation modulates the capillary endothelial glycocalyx. Circ Res 2004; 94: 77-82.

34. Cramer T, Johnson RS. A novel role for the hypoxia inducible transcription factor HIF-1alpha: Critical regulation of inflammatory cell function. Cell Cycle 2003; 2: 192-3.

35. Kong $\mathrm{T}$, Eltzschig $\mathrm{HK}$, Karhausen $\mathrm{J}$ et al. Leukocyte adhesion during hypoxia is mediated by HIF-1-dependent induction of beta2 integrin gene expression. Proc Natl Acad Sci USA 2004; 101: 10440-5.

36. Tamura DY, Moore EE, Partrick DA et al. Acute hypoxemia in humans enhances the neutrophil inflammatory response. Shock 2002; 17: 269-73.

37. Colgan SP, Dzus AL, Parkos CA. Epithelial exposure to hypoxia modulates neutrophil transepithelial migration. J Exp Med 1996; 184: $1003-15$.

38. Rui T, Cepinskas G, Feng Q et al. Cardiac myocytes exposed to anoxia-reoxygenation promote neutrophil transendothelial migration. Am J Physiol, Heart Circ Physiol 2001; 281: H440-7.

39. Madara JL. Regulation of the movement of solutes across tight junctions. Annu Rev Physiol 1998; 60: 143-59.

40. Luscinskas FW, Ma S, Nusrat A et al. Leukocyte transendothelial migration: A junctional affair. Semin Immunol 2002; 14: 105-13.

41. Luscinskas FW, Ma S, Nusrat A et al. The role of endothelial cell 
lateral junctions during leukocyte trafficking. Immunol Rev 2002; 186: 57-67.

42. Carpenter TC, Stenmark KR. Hypoxia decreases lung neprilysin expression and increases pulmonary vascular leak. Am J Physiol, Lung Cell Mol Physiol 2001; 281: L941-8.

43. Schoch HJ, Fischer S, Marti HH. Hypoxia-induced vascular endothelial growth factor expression causes vascular leakage in the brain. Brain 2002; 125: 2549-57.

44. Karhausen JO, Furuta GT, Tomaszewski JE et al. Epithelial hypoxia-inducible factor-1 is protective in murine experimental colitis. J Clin Invest 2004; 114: 1098-106.

45. Rippe B, Haraldsson B. Transport of macromolecules across microvascular walls: The two-pore theory. Physiol Rev 1994; 74: 163-219.

46. Lum H, Malik AB. Regulation of vascular endothelial barrier function. Am J Physiol 1994; 267: L223-41.

47. Eltzschig HK, Thompson LF, Karhausen $J$ et al. Endogenous adenosine produced during hypoxia attenuates neutrophil accumulation: Coordination by extracellular nucleotide metabolism. Blood 2004; 104: 3986-92.

48. Kloner RA, Ellis SG, Lange R, Braunwald E. Studies of experimental coronary artery reperfusion: Effects on infarct size, myocardial function, biochemistry, ultrastructure and microvascular damage. Circulation 1983; 68(Suppl I): 8-15.

49. Kloner RA, Ganote CE, Jennings RB. The "no-reflow" phenomenon after temporary coronary occlusion in the dog. J Clin Invest 1974; 54: 1496-508

50. Maier RV, Bulger EM. Endothelial changes after shock and injury. New Horizons 1996; 4: 211-23.

51. Waxman K. Shock: Ischemia, reperfusion and inflammation. New Horizons 1996; 4: 153-60.

52. Gautam N, Olofsson AM, Herwald $\mathrm{H}$ et al. Heparin-binding protein (HBP/CAP37): A missing link in neutrophil-evoked alteration of vascular permeability. Nat Med 2001; 7: 1123-7.

53. Pereira HA. CAP37, a neutrophil-derived multifunctional inflammatory mediator. J Leukoc Biol 1995; 57: 805-12.

54. Lee SY, Madan A, Furuta GT et al. Lactase gene transcription is activated in response to hypoxia in intestinal epithelial cells. Mol Genet Metab 2002; 75: 65-9.

55. Hayashi M, Kim SW, Imanaka-Yoshida $\mathrm{K}$ et al. Targeted deletion of BMK1/ERK5 in adult mice perturbs vascular integrity and leads to endothelial failure. J Clin Invest 2004; 113: 1138-48.

56. Webb AR. Capillary leak. Pathogenesis and treatment. Minerva Anestesiol 2000; 66: 255-63.

57. Michel CC, Curry FE. Microvascular permeability. Physiol Rev 1999; 79: 703-61.

58. Baxter GF. Role of adenosine in delayed preconditioning of myocardium. Cardiovasc Res 2002; 55: 483-94.

59. Mubagwa K, Flameng W. Adenosine, adenosine receptors and myocardial protection: An updated overview. Cardiovasc Res 2001; 52: 25-39.

60. Linden J. Molecular approach to adenosine receptors: Receptormediated mechanisms of tissue protection. Annu Rev Pharmacol Toxicol 2001; 41: 775-87.

61. Hasko G, Cronstein BN. Adenosine: An endogenous regulator of innate immunity. Trends Immunol 2004; 25: 33-9.

62. McCallion K, Harkin DW, Gardiner KR. Role of adenosine in immunomodulation: Review of the literature. Crit Care Med 2004 32: $273-7$.

63. Sitkovsky MV, Lukashev D, Apasov S et al. Physiological control of immune response and inflammatory tissue damage by hypoxiainducible factors and adenosine A2A receptors. Annu Rev Immunol 2004; 22: 657-82

64. Ohta A, Sitkovsky M. Role of G-protein-coupled adenosine receptors in downregulation of inflammation and protection from tissue damage. Nature 2001; 414: 916-20.

65. Hochachka PW, Lutz PL. Mechanism, origin, and evolution of anoxia tolerance in animals. Comp Biochem Physiol, Part B Biochem Mol Biol 2001; 130: 435-59.

66. O'Farrell PH. Conserved responses to oxygen deprivation. J Clin Invest 2001; 107: 671-4.
67. Boutilier RG. Mechanisms of cell survival in hypoxia and hypothermia. J Exp Biol 2001; 204: 3171-81.

68. Marcus AJ, Broekman MJ, Drosopoulos JH et al. Metabolic control of excessive extracellular nucleotide accumulation by CD39/ ecto-nucleotidase-1: Implications for ischemic vascular diseases. J Pharmacol Exp Ther 2003; 305: 9-16.

69. Robson SC. Thromboregulation by endothelial cells: Significance for occlusive vascular diseases. Arterioscler Thromb Vasc Biol 2001; 21: 1251-2

70. Robson SC, Kaczmarek E, Siegel JB et al. Loss of ATP diphosphohydrolase activity with endothelial cell activation. J Exp Med 1997; 185: 153-63.

71. Marcus AJ, Broekman MJ, Drosopoulos JH et al. The endothelial cell ecto-ADPase responsible for inhibition of platelet function is CD39. J Clin Invest 1997; 99: 1351-60.

72. Imai M, Takigami K, Guckelberger $\mathrm{O}$ et al. Modulation of nucleoside [correction of nucleotide] triphosphate diphosphohydrolase-1 (NTPDase-1)cd39 in xenograft rejection. Mol Med 1999; 5: 743-52.

73. Gayle RB III, Maliszewski CR, Gimpel SD et al. Inhibition of platelet function by recombinant soluble ecto-ADPase/CD39. J Clin Invest 1998; 101: 1851-9.

74. Koyamada N, Miyatake T, Candinas D et al. Apyrase administration prolongs discordant xenograft survival. Transplantation 1996; 62: 1739-43.

75. Pinsky DJ, Broekman MJ, Peschon JJ et al. Elucidation of the thromboregulatory role of $\mathrm{CD} 39$ /ectoapyrase in the ischemic brain. J Clin Invest 2002; 109: 1031-40.

76. Enjyoji K, Sevigny J, Lin Y et al. Targeted disruption of cd39/ATP diphosphohydrolase results in disordered hemostasis and thromboregulation. Nat Med 1999; 5: 1010-7.

77. Fabre JE, Nguyen M, Latour A et al. Decreased platelet aggregation, increased bleeding time and resistance to thromboembolism in P2Y1-deficient mice. Nat Med 1999; 5: 1199-202.

78. Napieralski R, Kempkes B, Gutensohn W. Evidence for coordinated induction and repression of ecto-5'-nucleotidase (CD73) and the A2a adenosine receptor in a human B cell line. Biol Chem 2003; 384: 483-7.

79. McDonald DM, Thurston G, Baluk P. Endothelial gaps as sites for plasma leakage in inflammation. Microcirculation 1999; 6: $7-22$.

80. Takano T, Clish CB, Gronert K et al. Neutrophil-mediated changes in vascular permeability are inhibited by topical application of aspirin-triggered 15-epi-lipoxin A4 and novel lipoxin B4 stable analogues. J Clin Invest 1998; 101: 819-26.

81. Thompson LF, Eltzschig HK, Ibla JC et al. Crucial role for ecto-5'nucleotidase (CD73) in vascular leak during hypoxia. J Exp Med 2004; 200: 1395-405.

82. Bear JE, Svitkina TM, Krause M et al. Antagonism between Ena/ VASP proteins and actin filament capping regulates fibroblast motility. Cell 2002; 109: 509-21.

83. Lawrence DW, Comerford KM, Colgan SP. Role of VASP in reestablishment of epithelial tight junction assembly after $\mathrm{Ca}^{2+}$ switch. Am J Physiol, Cell Physiol 2002; 282: C1235-45.

84. Ogawa S, Koga S, Kuwabara K et al. Hypoxia-induced increased permeability of endothelial monolayers occurs through lowering of cellular cAMP levels. Am J Physiol 1992; 262: C546-54.

85. Ogawa S, Shreeniwas R, Butura C et al. Modulation of endothelial barrier function by hypoxia: Perturbation of barrier and anticoagulant function, and induction of a novel factor $\mathrm{X}$ activator. Adv Exp Med Biol 1990; 281: 303-12

86. Kokura S, Yoshida N, Yoshikawa T. Anoxia/reoxygenationinduced leukocyte-endothelial cell interactions. Free Radic Biol Med 2002; 33: 427-32.

87. Haddad JJ. Science review: Redox and oxygen-sensitive transcription factors in the regulation of oxidant-mediated lung injury: Role for hypoxia-inducible factor-1alpha. Crit Care 2003; 7: 47-54

88. Saadi S, Wrenshall LE, Platt JL. Regional manifestations and control of the immune system. FASEB J 2003; 16: 849-56.

89. Johnson-Leger C, Aurrand-Lions M, Imhof BA. The parting of the endothelium: Miracle, or simply a junctional affair? J Cell Sci 2000; 113: 921-33 
90. Semenza GL. HIF-1, O(2), and the 3 PHDs: How animal cells signal hypoxia to the nucleus. Cell 2001; 107: 1-3.

91. Welbourne CRB, Goldman G, Valeri CR et al. Pathophysiology of ischaemia reperfusion injury: Central role of the neutrophil. $\mathrm{Br} \mathrm{J}$ Surg 1991; 78: 651-5.

92. Novak I. ATP as a signaling molecule: The exocrine focus. News Physiol Sci 2003; 18: 12-7.

93. Burnstock G. Potential therapeutic targets in the rapidly expanding field of purinergic signalling. Clin Med 2002; 2: 45-53.

94. Wang TF, Guidotti G. CD39 is an ecto- $\left(\mathrm{Ca}^{2+}, \mathrm{Mg}^{2+}\right)$-apyrase. $\mathrm{J}$ Biol Chem 1996; 271: 9898-901.

95. Mizumoto N, Kumamoto T, Robson SC et al. CD39 is the dominant Langerhans cell-associated ecto-NTPDase: Modulatory roles in inflammation and immune responsiveness. Nat Med 2002; 8: 358-65.

96. Gendron FP, Benrezzak O, Krugh BW et al. Purine signaling and potential new therapeutic approach: Possible outcomes of NTPDase inhibition. Curr Drug Targets 2002; 3: 229-45.

97. Qawi I, Robson SC. New developments in anti-platelet therapies: Potential use of CD39/vascular ATP diphosphohydrolase in thrombotic disorders. Curr Drug Targets 2000; 1: 285-96.

98. Gangadharan SP, Imai M, Rhynhart KK et al. Targeting platelet aggregation: CD39 gene transfer augments nucleoside triphosphate diphosphohydrolase activity in injured rabbit arteries. Surgery 2001; 130: 296-303.

99. Goepfert C, Imai M, Brouard S et al. CD39 modulates endothelial cell activation and apoptosis. Mol Med 2000; 6: 591-603.

100. Imai $\mathrm{M}$, Takigami $\mathrm{K}$, Guckelberger $\mathrm{O}$ et al. Recombinant adenoviral mediated CD39 gene transfer prolongs cardiac xenograft survival. Transplantation 2000; 70: 864-70.

101. Madara JL, Patapoff TW, Gillece-Castro B et al. $5^{\prime}$-adenosine monophosphate is the neutrophil-derived paracrine factor that elicits chloride secretion from T84 intestinal epithelial cell monolayers. J Clin Invest 1993; 91: 2320-5.

102. Airas L, Hellman J, Salmi M et al. CD73 is involved in lymphocyte binding to the endothelium: Characterization of lymphocytevascular adhesion protein 2 identifies it as CD73. J Exp Med 1995; 182: 1603-8.

103. Resta R, Hooker SW, Laurent AB et al. Insights into thymic purine metabolism and adenosine deaminase deficiency revealed by transgenic mice overexpressing ecto- $5^{\prime}$-nucleotidase (CD73). J Clin Invest 1997; 99: 676-83.

104. Gnaiger E. Bioenergetics at low oxygen: Dependence of respiration and phosphorylation on oxygen and adenosine diphosphate supply. Respir Physiol 2001; 128: 277-97.

105. Saito H, Nishimura M, Shinano $H$ et al. Plasma concentration of adenosine during normoxia and moderate hypoxia in humans. Am J Respir Crit Care Med 1999; 159: 1014-8.

106. Mo FM, Ballard HJ. The effect of systemic hypoxia on interstitial and blood adenosine, AMP, ADP and ATP in dog skeletal muscle. J Physiol 2001; 536: 593-603.

107. Edmunds NJ, Marshall JM. The roles of nitric oxide in dilating proximal and terminal arterioles of skeletal muscle during systemic hypoxia. J Vasc Res 2003; 40: 68-76.

108. Ray CJ, Abbas MR, Coney AM, Marshall JM. Interactions of adenosine, prostaglandins and nitric oxide in hypoxia-induced vasodilatation: In vivo and in vitro studies. J Physiol 2002; 544: 195-209.

109. Bryan PT, Marshall JM. Cellular mechanisms by which adenosine induces vasodilatation in rat skeletal muscle: Significance for systemic hypoxia. J Physiol 1999; 514: 163-75.

110. Revan S, Montesinos MC, Naime D et al. Adenosine A2 receptor occupancy regulates stimulated neutrophil function via activation of a serine/threonine protein phosphatase. J Biol Chem 1996; 271: 17114-8.
111. Chaudary N, Naydenova Z, Shuralyova I, Coe IR. Hypoxia regulates the adenosine transporter, $\mathrm{mENT} 1$, in the murine cardiomyocyte cell line, HL-1. Cardiovasc Res 2004; 61: 780-8.

112. Ji X, Kim YC, Ahern DG et al. [3H]MRS 1754, a selective antagonist radioligand for $\mathrm{A}(2 \mathrm{~B})$ adenosine receptors. Biochem Pharmacol 2001; 61: 657-63.

113. Di Virgilio F, Chiozzi P, Ferrari D et al. Nucleotide receptors: An emerging family of regulatory molecules in blood cells. Blood 2001; 97: 587-600.

114. Hollopeter G, Jantzen HM, Vincent D et al. Identification of the platelet ADP receptor targeted by antithrombotic drugs. Nature 2001; 409: 202-7.

115. Communi D, Janssens R, Suarez-Huerta $\mathrm{N}$ et al. Advances in signalling by extracellular nucleotides. The role and transduction mechanisms of P2Y receptors. Cell Signal 2000; 12: 351-60.

116. Abbracchio MP, Boeynaems JM, Barnard EA et al. Characterization of the UDP-glucose receptor (re-named here the P2Y14 receptor) adds diversity to the $\mathrm{P} 2 \mathrm{Y}$ receptor family. Trends Pharmacol Sci 2003; 24: 52-5.

117. Humphreys BD, Virginio C, Surprenant A et al. Isoquinolines as antagonists of the P2X7 nucleotide receptor: High selectivity for the human versus rat receptor homologues. Mol Pharmacol 1998; 54: 22-32.

118. Burnstock G. Purinergic signaling and vascular cell proliferation and death. Arterioscler Thromb Vasc Biol 2002; 22: 364-73.

119. Di Virgilio F, Baricordi OR, Romagnoli R, Baraldi PG. Leukocyte P2 receptors: A novel target for anti-inflammatory and anti-tumor therapy. Curr Drug Targets Cardiovasc Haematol Disord 2005; 5: 85-99.

120. Inbe $\mathrm{H}$, Watanabe $\mathrm{S}$, Miyawaki $\mathrm{M}$ et al. Identification and characterization of a cell-surface receptor, P2Y15, for AMP and adenosine. J Biol Chem 2004; 279: 19790-9.

121. He W, Miao FJ, Lin DC et al. Citric acid cycle intermediates as ligands for orphan G-protein-coupled receptors. Nature 2004; 429: 188-93.

122. Qi AD, Harden TK, Nicholas RA. GPR980/99, proposed to be the P2Y15 receptor activated by adenosine and AMP, is not a P2Y receptor. Purinergic Signal 2004; 1: 67-74.

123. Abbracchio MP, Burnstock G, Boeynaems JM et al. The recently deorphanized GPR80 (GPR99) proposed to be the P2Y15 receptor is not a genuine P2Y receptor. Trends Pharmacol Sci 2005; 26: 8-9.

124. Hasko G, Sitkovsky MV, Szabo C. Immunomodulatory and neuroprotective effects of inosine. Trends Pharmacol Sci 2004; 25: 152-7.

125. Jin X, Shepherd RK, Duling BR, Linden J. Inosine binds to A3 adenosine receptors and stimulates mast cell degranulation. J Clin Invest 1997; 100: 2849-57.

126. Tilley SL, Wagoner VA, Salvatore CA et al. Adenosine and inosine increase cutaneous vasopermeability by activating A(3) receptors on mast cells. J Clin Invest 2000; 105: 361-7.

127. Gomez G, Sitkovsky MV. Differential requirement for A2a and A3 adenosine receptors for the protective effect of inosine in vivo. Blood 2003; 102: 4472-8. Epub 2003 Aug 4428.

128. Hasko G, Kuhel DG, Nemeth ZH et al. Inosine inhibits inflammatory cytokine production by a posttranscriptional mechanism and protects against endotoxin-induced shock. J Immunol 2000; 164: 1013-9.

129. Ware LB, Matthay MA. The acute respiratory distress syndrome. N Engl J Med 2000; 342: 1334-49.

130. Hotchkiss RS, Karl IE. The pathophysiology and treatment of sepsis. N Engl J Med 2003; 348: 138-50.

131. Karhausen J, Haase VH, Colgan SP. Inflammatory hypoxia: Role of hypoxia-inducible factor. Cell Cycle 2005; 4: 256-8.

132. Cohen ES, Law WR, Easington CR et al. Adenosine deaminase inhibition attenuates microvascular dysfunction and improves survival in sepsis. Am J Respir Crit Care Med 2002; 166: 16-20. 